\title{
ANAESTHESIA IN GENERAL PRACTICE
}

\author{
This is one of a series of articles, contributed by invitation
}

\section{RISKS OF ANAESTHESIA-II \\ BY \\ M. D. NOSWORTHY, M.D., B.Ch., D.A. Respiratory Obstruction}

The maintenance of a free and adequate respiratory exchange is of the first importance. Respiratory obstruction occurs when the patient's respiratory efforts do not run parallel with the gaseous interchange. An obstructed airway dams back carbon dioxide in the lungs, hampers the entry of oxygen, exhausts the patient through his muscular efforts to get air in, embarrasses the circulation, and may precipitate a vascular accident. When examining the patient beforehand the anaesthetist can often foresee whether he may have obstructive or other difficulties. Questioning the patient about his normal activities - does he get out of breath after climbing stairs, etc.?-is a better guide to his physical fitness than any formula. The presence of anaemia or dehydration should also be looked for; it is obviously of advantage to know in advance whether fluids are likely to be required during the operation. The full-blooded, short-necked type of individual is prone to become congested and obstructed during induction. The condition of the teeth and the patency of the nostrils are worthy of examination. Both the pharynx and the larynx of the heavy smoker are very irritable, and it is useful to remember that when morphine and basal hypnotics depress the sensitivity of the respiratory centre they do not decrease the sensitivity of the larynx to the same extent. This combination of circumstances does not facilitate induction with irritant anaesthetic agents.

It is most important to be certain that the stomach is empty before induction, and it must be borne in mind that fear markedly prolongs its emptying time. At a depth of anaesthesia sufficiently light for the occurrence of active vomiting, the laryngeal reflex is intact, so that the glottis will close. The patient, however, can only hold his breath until the alveolar content of carbon dioxide has risen to a level which, as it were, breaks through this reflex, and, if aspiration is to be avoided, it is imperative that the head should be lowered and any vomit in the pharynx removed before this breath is taken. Gastric and obstetrical emergency cases should have a preliminary wash-out if there is any likelihood of the stomach not being empty. The manipulations of the obstetrician during an external version may well express gastric contents, and at the depth of anaesthesia necessary for this manœuvre the laryngeal reflex will have been abolished. Should vomiting occur there must be no delay in tipping the patient head downwards and removing the vomit, preferably with suction. The anaesthetist must also remember the possibility of reversed peristalsis. A patient suffering from intestinal obstruction must be expected to regurgitate copious quantities of intestinal contents at any moment.

\section{SPECIAL CONSIDERATIONS}

Before embarking on an operation on the nose, mouth, or throat the anaesthetist must decide which of the various methods he is going to adopt to safeguard the patient from the aspiration of blood or pus. Apparatus must be tested before being used to see that there is no possibility of blowing liquid ether into the patient's lungs. During and after operation the anaesthetist must bear in mind the possibility of a foreign body, such as a forgotten swab, obstructing the air passages; a suitable posture must also be maintained during recovery. Some oedema of the glottis may be expected as a result of inflammatory processes in the pharynx or neck. This will become much worse if any asphyxia accompanies induction. Tracheotomy may be advisable before the induction of anaesthesia, and this is the last resort for any complete obstruction situated in or above the glottis. It is inadvisable to give any general anaesthetic before opening a quinsy. It is to be expected that a patient with carcinoma of the oesophagus will regurgitate a quantity of mucus.

Thyroid patients with obstruction should be given little premedication unless the anaesthetist is on the spot, as their obstruction often becomes worse when they go to sleep. When the obstruction is very severe intubation under local anaesthesia is a wise precaution. Bilateral abductor paralysis of the cords or tracheal collapse will necessitate immediate intubation. The presence of emphysema or of secretion in the tracheo-bronchial tree interferes with free gaseous interchange; it takes longer to get oxygen and anaesthetic agents into these patients, and, what is equally important, it takes longer than usual to get carbon dioxide and the anaesthetic out again.

The consequences of opening the pleural cavity require the anaesthetist's attention; if both cavities are opened artificial respiration with positive pressure is essential. The changing of the position of a patient too lightly anaesthetized, or the presenting of too concentrated an anaes. thetic vapour may also upset the respiratory rhythm. The angle of his head in relation to his neck and the position in which he lies during operation may interfere with normal respiratory exchange. Other points which may not be appreciated are that the use of either the kidney rest or the gall-bladder bridge is conducive to pulmonary collapse, and that the prone position and the weight of the abdominal contents on the diaphragm in the full Trendelenburg position hamper respiration.

\section{CONGESTED ASPHYXIAL OBSTRUCTION}

This may occur during induction. A rubber nasopharyngeal tube is useful if attempts to push the jaw forward fail. The tube must not be too large, and it must be passed before the congestion becomes marked, otherwise bleeding will follow and make matters far worse. The insertion of the usual pharyngeal airway when the spasm is fully developed is well-nigh impossible. As his alveolar content of carbon dioxide rises the patient's efforts to get air in are usually successful Oxygen is what he needs, and an attempt must be made to force this in; for unless the asphyxia is relieved the muscular spasm will not relax until shortly before death. When this relaxation occurs there is nearly always timethough it must not be counted upon-to insert an airway quickly and inflate the lungs with oxygen.

\section{ATONIC OBSTRUCTION}

For this type of respiratory obstruction, which develops when the muscles of the jaw, tongue, and pharynx have lost their tone, a pharyngeal airway is indicated. Waters pattern is superior to any other, and if the base of the 
tongue is pressed forward with the left forefinger so.that the airway drops into place it is non-traumatic, in spite of being made of metal. This airway can be inserted

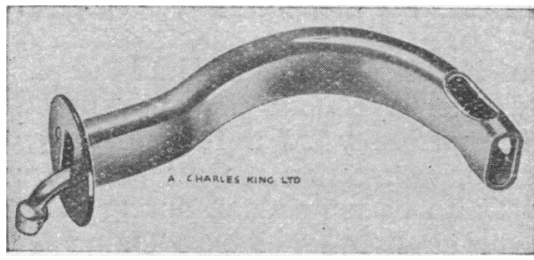

Waters' Pharyngeal Airway.

even when the jaws are in spasm (see above) if the left forefinger is used as a wedge behind the last molar tooth.

\section{LARYNGEAL SPASM AND STRIDOR}

These are two degrees of the same reflex. The appearance of either during induction shows that the anaesthetic vapour is too strong, or that particles of mucus are directly irritating the vocal cords. Surgical stimulation, especially of the peritoneum or perineal region, will set up these reflexes if the depth of anaesthesia is insufficient to dull them. A low-pitched stridor usually passes off as anaesthesia is deepened. With a high-pitched stridor, however, the cords practically close during inspiration and the respiratory exchange is seriously reduced. If, when all surgical intervention is stopped, the cords relax the anaesthetist may be able to regain control. As a rule, however, the reflex-once thoroughly irritated-is likely to recur at the slightest provocation, even though anaesthesia is subsequently maintained at a much deeper level than would have been necessary had it been adequate in the first instance.

Intubation as a cure for laryngeal stridor is a confession of failure, but it is better to do this than to allow the respiratory obstruction to remain throughout the operation. A tube a little stiffer and smaller than usual should be employed, and it should be introduced-in contrast to the normal practice-during expiration. Intubation at too light a depth of anaesthesia may make matters worse for a time, as the patient may hold his breath and keep his chest rigid and immobile long enough for his circulation to become embarrassed. While on the subject of intubaticn, I must add that the anaesthetist will save himself needless worry if he makes certain before the operation starts that the tube and connexions cannot come adrift, and that there is no chance of the patient biting through the tube. The lumen of a soft tube can easily be obliterated by kinking or by pressure from a throat pack. Finally, care must be taken not to introduce the tube so far that its end stretches beyond the bifurcation of the trachea into one bronchus.

\section{Primary Cardiac Failure}

Although I have never seen the condition, many cases have been reported in which the combination of light anaesthesia and excess of adrenaline in the blood stream as a result of fear, excitement, or the introduction of the drug itself, together with the stimulation of operative intervention, has resulted in ventricular fibrillation. In my opinion the commonest cause of cardiac arrest under chloroform anaesthesia is a relative overdose following the sudden inspiration of a large quantity of the anaesthetic. For example, this might occur during the deep breaths following a period of apnoea or shallow breathing, the deep breaths of the second stage of anaesthesia (especially if the heart is already embarrassed by some degree of asphyxia), and those following a cough, or, if anaesthesia is incomplete, the first incision. In such an emergency a child may be held up by his feet, or in the case of an adult the table may be tilted into the full Trendelenburg position at once. Artificial respiration must be started by rhythmical squeezing of a bag filled with oxygen. Local stimulation, in the form of hot cloths, may be applied to the praecordium. If the patient's condition does not improve in a minute or two cardiac puncture through the third right intercostal space, followed by cardiac massage, must be resorted to, artificial respiration being carried on the whole time.

\section{Circulatory Depression}

Collapse from cerebral anaemia may be brought about by a sudden change from the Trendelenburg to the horizontal position, or by allowing a patient who is deeply anaesthetized, or under the influence of a spinal anaesthetic, to be propped up. Many factors embarrass the circulation and contribute to its exhaustion during operation. The adoption of unnatural postures; the introduction of abdominal packs; the presence of respiratory obstruction; the inadequacy of the anaesthesia for the operative procedure in hand; the loss of muscle tone, the abolition of normal chest movements, and the toxic effects of the anaesthetic agent in prolonged deep anaesthesia ; surgical trauma to muscle and nervous tissue; and loss of body temperature and fluids may all play a part in the production of shock. It is a good practice to introduce rectal or subcutaneous salines throughout long operations which are known to be associated with shock. A pause for recovery in the middle of a severe operation will often enable a patient to rally and then withstand a fresh onslaught. The patient usually " compensates" for a time when haemorrhage occurs, and circulatory failure sets in later if this compensation is overtaxed. All degrees of circulatory depression are met with. The onset of true shock is insidious, and the classical signs are well known. If repeated observations of the blood pressure and pulse rate are made treatment can be started before this advanced state is reached, and results will be much more satisfactory.

The table should be slightly tilted head downwards, the patency of the airway should be verified, and an adequate respiratory exchange kept up with an atmosphere rich in oxygen. The lightest possible level of anaesthesia should be maintained-if an inadequate depth of anaesthesia was primarily responsible, it is too late to do any good by increasing the depth after the appearance of true shock. A 5 per cent. solution of glucose should be given intravenously in sufficient quantity to bring the systolic blood pressure up to 90 per cent. of the patient's normal figure. A blood transfusion may be advisable. After operation the patient must be kept warm but not over-heated. Oxygen should be delivered at a rate of six to eight litres a minute through a nasal catheter, the perforated tip of which should protrude as deeply into the oropharynx as possible without giving rise to swallowing movements; this is especially indicated if there is any restlessness. Although morphine, by lowering metabolism, reduces the patient's requirements of oxygen, it also reduces his oxygen intake by decreasing his respiratory exchange at the same time. Unless the patient's need is urgent fluid is best introduced by mouth or rectum, because, among other reasons, he can then reject any excess.

\section{Convulsions}

"Jactitations" under nitrous oxide are asphyxial in origin and call for the introduction of air or oxygen. So-called "ether" convulsions usually occur when the temperature is high in patients with some infection 
-for example, a sick child with acute appendicitis. They begin with twitchings in the muscles of the face, and may soon spread and become generalized. The breathing has usually been depressed or somewhat obstructed, and it is thought that the raised alveolar content of carbon dioxide is the factor which determines the onset of the convulsions in most cases. In support of this hypothesis it may be mentioned that convulsions have begun upon the addition of carbon dioxide to facilitate blind intubation, stopping when the gas was turned off, and not returning although ether was used throughout the operation. Whatever the stage of the operation the anaesthetic must be discontinued until the convulsions are controlled. The respiratory exchange must be increased by rhythmical squeezing of a bag filled with oxygen, though this may be difficult to carry out on account of the respiratory obstruction. If no improvement follows lowering the alveolar content of carbon dioxide, sufficient evipan or pentothal should be injected intravenously to control the convulsions, the artificial respiration being meanwhile continued.

Convulsions-and circulatory collapse-are the most serious manifestations of poisoning by cocaine or allied drugs. Other symptoms and signs of toxicity have as a rule been missed or unheeded. These convulsions will be stopped by the intravenous injection of a barbiturate, but, as these patients all die of oxygen want, it is the anaesthetist's duty to start artificial respiration without delay, and to maintain an efficient respiratory exchange while the barbiturate solution is being prepared and injected.

\section{Other Risks}

Damage to the Eyes.-Precautions must be taken to avoid any anaesthetic agent getting into the eyes. The face-piece or gamgee tissue and the anaesthetist's fingers must not rub them. The eyes should be kept closed throughout the operation. If they tend to open they must be repeatedly moistened by the moving up and down of the upper lids. If the reflexes have not returned by the end of the operation and the eyes tend to remain partly open, a drop of castor oil should be instilled into each eye, and the patient should be so arranged in bed that he cannot rub his eyes on the pillow during recovery.

Explosions.-Sources of explosion are: the cautery, naked flames and fires, diathermy machines and electric motors, faulty electrical connexions, and static electricity. In the concentrations used during anaesthesia the only agents which are non-explosive are basal hypnotics, local anaesthetics, nitrous-oxide-oxygen, carbon dioxide, and chloroform. The quarter from which any draught in the theatre is coming and the relative weights of the anaesthetic and air will determine in which direction the vapour will travel. Appreciation of these points may, in certain circumstances, permit the use of the diathermy needle in the presence of explosive mixtures. Static electricity is fortunately an uncommon factor in this country, a possible explanation for this being the high relative humidity of the atmosphere. A wise precaution is to damp the inside of the bag, rubber tubing, and face-piece with water before induction, thereby reducing friction and the risk of electric sparks. Soon after the anaesthetic is begun complete saturation of the atmosphere takes place in a closed system with carbon dioxide absorption, making the risk of explosion from this cause, for practical purposes, negligible. In the absence of leakage from such a closed system in which the anaesthetic mixture is explosive the diathermy needle may be used with safety except in the vicinity of the lungs or air passages.

\section{MEDICAL USES OF RADIUM REPORT BY MEDICAL RESEARCH COUNCIL}

The report of the Medical Research Council on the medical uses of radium during the year $1936^{1}$ illustrates very strikingly the increased use of radiotherapeutic measures in the treatment of malignant disease. When the annual figures contributed by the various centres during the years 1930-6 are added together a total of 18,496 cases of malignant disease is reached, of which 5,452 (29.5 per cent.) were treated by surgery alone, and 7,355 (39.8 per cent.) by radium alone.

The numbers of cases treated by different methods during the year 1936 are shown in the accompanying table.

TABLE I.-Relative Frequency of Surgical and Radiotherapeutic Treatments in 1936

\begin{tabular}{|c|c|c|c|c|c|}
\hline & & & & $\begin{array}{c}\text { Number Treated } \\
\text { in } 1936\end{array}$ & Percentage \\
\hline By all methods $\ldots$ & . & .. & .. & 4,419 & 100.0 \\
\hline , surgery alone .. & . & $\cdots$ & $\ldots$ & 1,365 & 30.6 \\
\hline ", radium alone* & . & -. & . & 1,426 & 32.3 \\
\hline , $x$ rays alone $\ldots$ & $\cdots$ & -. & $\cdots$ & 858 & 19.5 \\
\hline " surgery + radium & $\cdots$ & $\cdots$ & . & 224 & 5.1 \\
\hline$" \quad, \quad+x$ rays & $\cdots$ & $\cdots$ & $\cdots$ & 285 & 6.5 \\
\hline , radium $+x$ rays & $\cdots$ & . & . & 215 & 4.8 \\
\hline ", surgery + radium & $x$ & .. & . & 46 & 1.0 \\
\hline
\end{tabular}

* Treatment by " radium alone" may involve surgery of access.

\section{Cancer of the Breast}

The Birmingham General Hospital presents a very full account of the work done in this condition during the past year. There has been a slight diminution in the number of cases treated by radium, which is attributable to the more extended use of high-voltage $x$-ray therapy in operable and border-line cases, and subsequent amputation, whenever this is practicable. When treatment by radium has been carried out the exact position of the needles with respect to each other, to the tumour, and to the skin has received careful attention; and, in addition to clinical measurements made at the time of insertion, valuable assistance has been afforded by combined radioscopy, radiography, and stereoscopy. The value of the last-named method is greatly increased with practice; but it is pointed out that these methods still leave a good deal to be desired, and some improved technique would be of great value, though it would have to be simple in application and at the same time suitable to the large numbers of needles used for treating carcinoma of the breast.

In treating axillary lymph glands it is found that, especially in stout patients, many receive an inadequate dose owing to the difficulty of securing even distribution of dosage. Attempts have therefore been made to use barrages from three or more planes of needles without placing any in the axilla itself. Stainless steel wire has entirely replaced silkworm gut for securing the needles, and the filtration has been increased to $0.9 \mathrm{~mm}$. platinum by the use of additional sheaths. At Birmingham, as elsewhere, surgery is advocated in all early cases, and the radiotherapist treats only such as are unsuitable for surgery owing to some accompanying general condition such as advanced age, diabetes, pulmonary or cardiac disease of long standing, and refusal of the patient to submit to operation. At the Marie Curie Hospital cases at the time unsuitable for operation have been treated during the past year by the low-intensity method of $x$-ray therapy.

St. Bartholomew's Hospital reports on the results of a routine histological examination of the breast and axillary

${ }^{1}$ Medical Uses of Radium. Summary of Reports from Research Centres for 1936. " Medical Research Council. Special Report Series, No. 226. H.M. Stationery Office. (1s.) 